\title{
LA GESTIÓN NO LITIGIOSA DE LOS CONFLICTOS JURÍDICO-PRIVADOS Y EL APRENDIZAJE DEL DERECHO CIVIL: LA SIMULACIÓN ${ }^{1}$
}

\author{
NON- LITIGIOUS MANAGEMENT OF LEGAL CONFLICTS AND \\ LEARNING OF CIVIL LAW: THE SIMULATION
}

\author{
Silvia Algaba Ros \\ Profesora de Derecho Civil \\ salgaba@uma.es \\ Universidad de Málaga
}

\begin{abstract}
Resumen:
El aprendizaje del Derecho debe tener como horizonte promover la cultura de la paz. Con este objetivo, se analizan las competencias que deben incorporarse al estudio de las asignaturas de derecho civil que se imparten en el Grado en Derecho a fin de favorecer que el estudiante incorpore en su futura actuación profesional destrezas que propicien la gestión consensual y constructiva de los conflictos jurídicos-privados.
\end{abstract}

En este contexto, la simulación se muestra como una estrategia docente idónea al permitir un aprendizaje significativo a través del cual el estudiante pueda familiarizarse con formas de gestión de conflicto no litigiosas y sea conocedor de las ventajas personales y sociales que su uso puede implicar.

Palabras clave: Aprendizaje del derecho civil, competencia de "negociación y conciliación", mediación, gestión consensuada de los conflictos jurídicos-privados, simulación.

\begin{abstract}
:
Learning law should promote a culture of peace. This paper analyzes the competences that should be incorporated into the study of civil law subjects in order to encourage a positive and peaceful conflict management. Simulation is a suitable strategy to achieve this objective.
\end{abstract}

Keywords: Learning civil law, negotiation capacity, conflict management, mediation, simulation.

Sumario: 1. El aprendizaje del Derecho civil y la gestión no litigiosa de los conflictos. 2. Competencias favorecedoras de la gestión consensuada en los conflictos jurídicociviles. 2.1. Capacidad de comprensión del conflicto. 2.2. Conocimiento del marco legal desde el acuerdo. 2.3. Capacidad para la gestión/resolución de los conflictos jurídicociviles de forma no litigiosa. 2.4. Capacidad de adoptar soluciones creativas. 3. La simulación como estrategia docente para favorecer la gestión consensual de los

\footnotetext{
${ }^{1}$ Este trabajo ha sido realizado en el ámbito de la Red Docente "La gestión de los conflictos jurídicos desde la colaboración. Estrategias docentes para la negociación y el acuerdo" (763/2018) y el Proyecto de Innovación Educativa "La simulación como herramienta de enseñanza-aprendizaje práctico en el ámbito jurídico" (PIE 17-112), ambos proyectos desarrollados en la Universidad de Málaga.

REJIE Nueva época: Revista Jurídica de Investigación e Innovación Educativa

Núm.20, Junio 2019, pp. 11-30

[En línea] http://www.revistas.uma.es/index.php/rejie

Recibido: noviembre 2018

Aceptado: enero 2019
} 
conflictos en las asignaturas de derecho civil. 3.1. Selección del conflicto. 3.2. Actividades formativas presenciales y no presenciales previas a la simulación. 3.3. Simulación. 3.4. Evaluación. 3.5. Reflexiones finales. Bibliografía.

\section{El aprendizaje del Derecho y la gestión no litigiosa de los conflictos.}

La incorporación al aprendizaje del Derecho de estrategias docentes favorecedoras de la gestión consensuada y pacífica de los conflictos es una exigencia actual pues una sociedad que gestione adecuadamente los conflictos jurídicos que se susciten en su seno, estará más preparada para abordar los desafíos del futuro.

Esta aspiración encuentra su justificación en una doble perspectiva: la función que debe cumplir un docente y las exigencias que plantean los nuevos derroteros que se van abriendo en el estudio de las distintas disciplinas jurídicas.

Efectivamente en el ámbito del Derecho, el fomento de la cultura de la paz es uno de los retos que se persiguen desde instancias jurídicas europeas y nacionales como se constata con la proliferación de normas que regulan la mediación y otros sistemas alternativos de gestión de conflictos ${ }^{2}$. Se trata de sistemas fundados en la colaboración, la negociación, la búsqueda de soluciones consensuadas donde los implicados superen el esquema ganar-perder. Desde el punto de vista del derecho civil, son numerosos los ejemplos existentes entre los que cabe señalar la Ley 5/2012 de 6 de julio de Mediación en asuntos civiles y mercantiles y la Ley $7 / 2017$ de 2 de noviembre relativa a la Resolución alternativa en materia de consumo. Pero también existen muestras de ello en otras disciplinas como el derecho del trabajo, derecho penal... aunque éstas escapan a nuestra reflexión. En cualquier caso, el camino que aspira a propiciar una cultura de la paz no implica alcanzar una sociedad sin conflictos pues de lo que se trata es de caminar hacia un horizonte donde existiendo el conflicto, consustancial a las relaciones humanas, la gestión del mismo sea constructiva.

También en el marco de la actividad del docente, se debe favorecer la expresada necesidad pues como se señala en la Estrategia de Educación de la UNESCO de 20142021 y en concreto en el Objetivo Estratégico $2^{\circ}$ : Empoderar a los educandos para que sean ciudadanos mundiales y creativos:

"El construir la paz en la mente de los hombres y las mujeres es central en el mandato de la UNESCO. En los próximos ocho años, la UNESCO ampliará considerablemente sus acciones para apoyar a los Estados Miembros en el desarrollo de sistemas educativos eficaces que ayuden a los educandos a adquirir los conocimientos, capacidades, valores, actitudes y comportamientos que se necesitan para forjar sociedades más pacíficas, inclusivas, equitativas y sostenibles".

A mayor abundamiento y en la misma línea, el RD 1393/2007, de 29 de octubre (en adelante, RD 1393/2007), por el que se establece la ordenación de las enseñanzas

\footnotetext{
2 Obsérvese que la gestión de los conflictos puede ser realizada por autotuleta, de forma heterocompositiva o autocompositiva. Señala MORENO CATENA (2017), que la autotutela puede ser entendida como aquel "medio por el cual el conflicto se resuelve mediante la imposición coactiva de la voluntad de uno de los sujetos sobre el otro", como son ejemplos los arts. 592 y 612 Cc. Señala el citado autor que la autocomposición es la "resolución del conflicto por las partes interesadas" y puede ser "unilateral o bilateral "dependiendo de si el resultado de la autocomposición se realiza a través de la actividad o inactividad de una de las partes o de ambas". Sin embargo, la resolución del conflicto es heterocompositiva cuando se impone por un tercero.
} 
universitarias oficiales, declara en su Preámbulo que “...Finalmente, se debe tener en cuenta que la formación en cualquier actividad profesional debe contribuir al conocimiento y desarrollo de los Derechos Humanos, los principios democráticos, los principios de igualdad entre mujeres y hombres, de solidaridad, de protección medioambiental, de accesibilidad universal y diseño para todos, y de fomento de la cultura de la paz".

Por todo ello, entendemos que es relevante favorecer el aprendizaje del estudiante en la gestión consensuada de los conflictos, pues va a implicar un manifiesto beneficio para la sociedad en general ahorrando costes económicos derivados de la excesiva litigiosidad y para la sociedad en particular reduciendo los costes personales que inciden en los sujetos afectados. Además, profesionalmente también tiene trascendencia pues el pleito que se gestiona adecuadamente es aquel en el que las partes no quedan definitivamente enfrentadas ni enemistadas.

Gestionar consensual y positivamente los conflictos implica utilizar estrategias profesionales que conecte el conocimiento y aplicación de las normas con el fomento de la autorresponsabilidad de las partes implicadas en el conflicto, favoreciendo la comunicación entre ellas, la empatía y el reconocimiento de sus intereses con el fin de permitir favorecer una gestión del conflicto que permita a ambas partes ganar.

Con los anteriores antecedentes y centrándonos en el ámbito del derecho civil, se realiza en este trabajo una propuesta de aprendizaje que tiene por objetivo familiarizar al estudiante con las formas de gestión positiva del conflicto y sus beneficios, pero con una visión realista que permita distinguir los casos en los que es viable. Por ello, no se trata de sustituir las estrategias propias de los sistemas confrontativos sino de sumar a las mismas otras que favorezcan la gestión consensuada de los conflictos. Se trata de sumar, no de restringir.

Coincidimos ZUMAQUERO GIL (2018, p.2) al afirmar que "cuando se trata de reflexionar acerca del modelo de enseñanza más adecuado para un determinado grupo de estudiantes, se debe partir de la idea que no todos los modelos de enseñanza permiten alcanzar los mismos objetivos". Ello nos lleva a tratar en este trabajo las competencias y estrategias docentes que permitan que el estudiante se familiarice con la resolución positiva, colaborativa y consensuada de los conflictos.

Si se pretende alcanzar una sociedad que favorezca la cultura de la paz, los futuros profesionales deben contar con competencias idóneas que permitan desarrollarla.

\section{Competencias favorecedoras de la gestión consensuada en los conflictos jurídico-civiles.}

Señala el citado RD 1393/2007 en su Preámbulo que el Espacio Europeo de Educación Superior (EEES) impulsó "un cambio en las metodologías docentes, que centra el objetivo en el proceso de aprendizaje del estudiante, en un contexto que se extiende ahora a lo largo de la vida... los planes de estudios conducentes a la obtención de un título deberán, por tanto, tener en el centro de sus objetivos la adquisición de competencias por parte de los estudiantes, ampliando, sin excluir, el tradicional enfoque basado en contenidos y horas lectivas". Pero como nos recuerda MESEGUER VELASCO (2009, p. 91) debemos partir del binomio "competencia-conocimiento pues "las competencias...necesitan para su aplicación conocimientos y los conocimientos, sin competencias no son suficientes en el contexto actual". 
En relación a las competencias, señala MANTILLA JÁCOME et al. (2016, p. 20) " $L a$ formación por competencias implica un cambio de paradigma, dejando atrás la enseñanza y entrando en el mundo del aprendizaje, restándole importancia a las metodologías de enseñanza que tenían como finalidad colmar de información al estudiante, mediante la impartición de cátedra magistral o la lectura de textos para una posterior verificación memorística; y a cambio de eso promover los procesos en los que el estudiante tenga la aptitud y habilidad para enfrentar una problemática por sí mismo y acuda a las fuentes del conocimiento para formular solución al problema". Además, en la planificación de las competencias se debe adoptar una postura dinámica pues como nos indica DE MIGUEL DÍAZ (2005, p. 34) "el crecimiento de un estudiante en una competencia dada es un proceso de naturaleza continua debido a las exigencias introducidas por el contexto, que cambia demandando nuevas respuestas". Sólo un aprendizaje significativo en competencias permitirá su continuo uso.

En el Libro Blanco del Título de Grado en Derecho de la $\operatorname{ANECA}^{3}$ (2005, p.61) se señalan que "los déficits formativos que afectan a la generalidad de los graduados universitarios españoles se concentran fundamentalmente en las competencias participativas..." entre las que resaltaba la de "negociación y de resolución de problemas." Y ello llevó a que en el citado Libro (2005, p.183) se incluyera "la capacidad de negociación y conciliación" en la lista de competencias y habilidades a fomentar en los futuros estudiantes de Derecho.

No obstante, transcurrido el tiempo, aunque es cierto que en algunos planes de estudios de Grado en Derecho de Universidades españolas esta competencia ha encontrado un desarrollo vertical materializándose en asignaturas concretas ${ }^{4}$, sin embargo, también existen planes de estudio en los que se hace referencia a la citada competencia sin encontrar un acomodo específico en los contenidos de las distintas asignaturas lo que incita a una profunda reflexión sobre la misma.

Aunque sería deseable la incorporación en los Planes del Grado en Derecho de asignaturas relacionadas con "el conflicto", "la negociación" o "la resolución de conflictos extrajudiciales", también es preciso implementar la competencia de "capacidad de negociación y conciliación" dada su excesiva generalidad si se aspira a su adecuada implementación.

Planteando el tema desde las asignaturas de derecho civil, si la finalidad perseguida es que los estudiantes estén preparados para afrontar decisiones profesionales desde la negociación y la colaboración en favor de una gestión consensuada, entonces la "competencia de negociación y conciliación" debería manifestarse a través de las siguientes competencias siempre desde la perspectiva del aprendizaje activo":

\footnotetext{
${ }^{3}$ Disponible en http://www.aneca.es/var/media/150240/libroblanco_derecho_def.pdf

${ }^{4}$ Es el caso del Grado en Derecho de la Universidad Pablo de Olavide en Sevilla que incorpora en sus planes de estudio una asignatura optativa transversal denominada "Métodos alternativos de resolución de conflictos" que tiene como descriptor "Arbitraje, mediación y conciliación y otros mecanismos para procesales de resolución de conflictos". Además en el Grado en Derecho incorpora como competencia básica: "capacidad de evitación y resolución de problemas". Consulta curso 2018-2019.

${ }^{5}$ En concreto señala MANTILLA JÁCOME et al. ( 2016, pp. 22 y 23) "Las metodologías activas de aprendizaje tiene por objeto construir el conocimiento desde la formulación de problemas, que incorporan la satisfacción de necesidades o solución de conflictos mediante el razonamiento y la creatividad; se ubica al estudiante de derecho en un aspecto del ejercicio social y racional del ser humano. En un tercer momento se realiza la reflexión jurídica aplicada al problema o conflicto desde el Derecho como instrumento social con el propósito de desarrollar la habilidad del estudiante para
} 
- Ser capaces de comprender la complejidad de los conflictos humanos en sus numerosas vertientes: sociales, jurídicas, personales, económicas...

- Conocer los diversos sistemas de gestión y resolución de conflictos que no tienen naturaleza heterocompositiva y comprender los beneficios de la gestión colaborativa y consensuada de los conflictos

- Comprender la trascendencia del conocimiento del marco legal que permite gestionar constructiva y consensuadamente los conflictos jurídico- civiles

- Estimular la creatividad para la gestión consensuada de los conflictos

\subsection{Capacidad de comprensión del conflicto.}

Los conflictos son habitualmente sentidos en la sociedad como negativos, sin embargo, cada vez va teniendo más predicamento la visión que ve el conflicto como necesario para el desarrollo de una sociedad democrática ${ }^{6}$. Como nos recuerda ALZATE SÁEZ DE HEREDIA (2008, pp. 22 y ss.) "Hoy en día... consideramos que el conflicto es un rasgo inevitable de las relaciones sociales. El problema estriba en que todo conflicto puede adoptar un curso constructivo o destructivo, y por lo tanto la cuestión no es tanto eliminar o prevenir el conflicto sino saber asumir dichas situaciones conflictivas y enfrentarnos a ellas con los recursos suficientes para que todos los implicados en dichas situaciones salgamos enriquecidos de ellas".

Entendemos que cuando el ciudadano recurre al Derecho mediante cualquiera de los operadores jurídicos, se encuentra ante un conflicto que es incapaz de gestionar y es importante que el profesional le ofrezca aquella estrategia que sea más adecuada a la tipología del conflicto y su contexto, siempre con el horizonte de favorecer la concordia y la cultura de la paz. Además, la cuestión es todavía más sangrante en algunos tipos de conflictos que son principalmente personales como ocurre con los familiares. Nos dice LÓPEZ Y LÓPEZ (1999, pp. 15 y 16) "Triste es el papel del Derecho cuando se topa con la familia. Orden jurídico y relación familiar mantienen una dialéctica a menudo imposible: cuando la familia se "juridifica" con frecuencia se han quebrado o extinguido los lazos de la estrecha relación de convivencia que la constituyen y se trata de restañar heridas incurables que han roto definitivamente la convivencia. Además, las normas legales reguladoras de las relaciones familiares gozan de una singular característica: su efectividad real para disciplinar la vida y economía de los miembros de la familia es muy dispar, pues el núcleo familiar resiste mal la injerencia de extraños sin quebrarse y porque las relaciones interpersonales familiares, más que leyes, prevalecen hábitos, roles definidos por la tradición, acuerdos tácitos entre sus miembros... En ese ámbito la ley cumple frecuentemente el papel de formulación de modelos, a menudo ilusorios y siempre ideales, y esta lejanía de la realidad termina convirtiendo el texto legal en poco más que en la expresión de un buen deseo".

Por todo ello es trascendente favorecer el conocimiento de la naturaleza de los $\operatorname{conflictos}^{7}$ por parte del estudiante para que pueda gestionarlos adecuadamente y

resolver problemas desde las normas jurídicas y su aplicación facultando para un futuro ejercicio profesional en el que pueda describir y prescribir situaciones jurídicas".

${ }^{6} \mathrm{La}$ democracia es uno de los valores esenciales de nuestro ordenamiento jurídico como se constata en el art. 1.1 de la Constitución Española: "España se constituye en un Estado social y democrático de Derecho..."

7 Afirma ALZATE (2008, p. 26) que siguiendo a MOORE y MAYER "los conflictos pueden ser de valores, estructurales, de intereses, de relaciones, de información o de comunicación" .Continúa el citado autor citando a BURTON que es preciso distinguir "tres tipos de motivaciones humanas: necesidades, valores e intereses. Las necesidades son motivaciones "universales y primordiales, y quizás genéticas"; 
siempre desde una "actitud constructiva". Afirma ZACCAGNINI $(2015, \text { p. 51) })^{8}$ que "Los conflictos se pueden plantear de forma alternativa como "problemas resolubles", y entonces su resolución resultará positiva. Ahora bien, para que esto ocurra debemos estar preparados y adoptar una actitud constructiva para su abordaje. Esta actitud supone, en primer lugar, asumir que los conflictos son algo normal y natural que concurre cotidianamente en las relaciones humanas. En segundo lugar, que son algo positivo, en la medida que nos avisan de un obstáculo dentro de una relación e importante para nosotros $y$, sobre todo, porque nos ayudan a mejorarla. $Y$, en tercer lugar, hay que contar con que los conflictos casi siempre son resolubles positivamente, llegando a un acuerdo mutuamente satisfactorio, con lo que mejoran las habilidades de relación y autoestima de las personas implicadas, reforzando la relación. Evidentemente... ésta debe ser la actitud... ante los conflictos. Pero para que se obtengan esos resultados es imprescindible, en primer lugar, estar preparados para identificarlos cuando aparezcan y abordarlos a tiempo. $Y$, en segundo lugar, afrontarlos, mediante la comunicación entre las partes implicadas"

Por lo expuesto, consideramos que se debe fomentar el conocimiento básico de las estructuras y tipos de conflicto analizando la distinción entre los intereses y posiciones de las partes. Asimismo, se debe capacitar al estudiante para detectar qué conflictos son idóneos para ser gestionados de forma constructiva. No podemos olvidar como ya nos recordaban GULLÓN BALLESTEROS y DÍEZ PICAZO $(2016$, pp. 150, 151) al analizar la interpretación de las normas jurídicas que, aunque en principio la interpretación jurídica es una interpretación que se enmarca exclusivamente en el ámbito de las normas siendo por tanto una "interpretación normativa", sin embargo "... no es posible llevar a cabo una tajante escisión entre los hechos y las normas, pues ésta está predeterminada por la valoración que a aquéllos se dé. No es posible, pues, confinar la interpretación al campo de lo estrictamente normativo, porque el supuesto de hecho previsto en la norma forma parte de ella, y el aplicador del Derecho ha de reconocer que el supuesto que se ha dado en realidad tiene un encaje en el previsto en la norma".

Sin afán exhaustivo, puede servir de referencia para identificar qué conflictos son los más idóneos para una gestión colaborativa y consensuada la "Guía práctica para la mediación intrajudicial publicada en la página del Consejo General del Poder Judicial" 9 donde se mencionan como supuestos especialmente idóneos para ser derivados a mediación "Cuando las partes mantengan lazos familiares o se perciba una evidente carga emocional... Cuando el conflicto surja en una relación continuada de las partes... Cuando la tutela del derecho suponga un coste no reembolsable (superior a la reclamación)... Todos aquellos en que existan entre las partes diversos litigios o se advierta que tras el actual seguirán otros... Cuando el cauce procesal aboque a un resultado de pérdida para ambas partes.... Cuando la decisión no permita adaptarse a

son necesarias para el desarrollo de la especie humana, y serán perseguibles por todos los medios disponibles. ... las necesidades son impulsos fundamentales compartidos por todos los organismos desarrollados. Ni cambian, como los valores e intereses, ni son escasos. Los valores son motivaciones que están culturalmente especificadas Son las costumbres y creencias peculiares de una cultura particular... Los intereses son motivaciones que cambian conforme a las circunstancias... Se relacionan, en general, con los roles sociales y los bienes materiales". La forma de abordar el conflicto dependerá de su tipología.

${ }^{8}$ Debe tenerse presente que el autor citado, ZACCAGNINI relaciona este párrafo con la figura del mediador, aunque en el trabajo se conecta con el estudiante.

9 http://www.poderjudicial.es/cgpj/es/Temas/Mediacion/Guia-para-la-practica-de-la-MediacionIntrajudicial/. Fecha de captura 21/03/2019 
los matices del caso, a las circunstancias... Si existe un alto nivel de incertidumbre en el resultado económico pretendido..."

En fin, con el desarrollo de esta capacidad se trata de que el estudiante que se enfrenta a un conflicto no sea un mero aplicador de la norma sino un gestor adecuado del conflicto teniendo presente las normas.

\subsection{Conocimiento del marco legal desde el acuerdo.}

Manifiesta PEÑUELAS Y REIXACH (2009, p. 75) que "La docencia práctica en nuestras Facultades debe ocuparse de las técnicas necesarias para resolver los problemas prácticos que plantea el funcionamiento del Derecho, entendido como sistema normativo imperfecto que se crea y aplica para configurar la vida social. Son esencialmente las técnicas de creación y las de aplicación del Derecho, en el sentido amplio de esa expresión: búsqueda de las normas vigentes y válidas aplicables a un determinado conflicto, interpretación de dichas normas; aplicación stricto sensu, integración del Derecho y argumentación jurídica, entre las más importantes". A todas ellas habría que añadir favorecer la búsqueda de la forma de resolución del conflicto más idónea en función de las circunstancias fomentado en lo posible el consenso. Con ello no se desatiende la necesidad del conocimiento del derecho civil pues la gestión positiva del conflicto debe necesariamente respetar ordenamiento jurídico y además precisa tener presente el estudio no confrontativo de las normas.

Efectivamente, se requiere un conocimiento del derecho civil que haga especial hincapié en cada institución en la trascendencia que tienen las normas imperativas y normas dispositivas que les son de aplicación

La necesidad de profundizar sobre la importancia de las normas imperativas se manifiesta en un triple sentido. En primer lugar, evitar que el profesional que utiliza estas estrategias quede sujeto a responsabilidad al haber favorecido en su caso una negociación que pueda ser ilegal. En segundo lugar, posibilitando un acuerdo que sea susceptible de ejecución. En tercer lugar, teniendo presente que como señala el art. 6.3Cc "los actos contrarios a las normas imperativas y a las prohibitivas son nulos de pleno derecho, salvo que en ellas se establezca un efecto distinto para el caso de contravención".

En cualquier caso, las normas imperativas permiten un cierto margen de actuación en la resolución de los conflictos pues como nos indica SOLETO MUÑOZ (2017, p. 28) citando al profesor MNOOKIN cabe negociar a la sombra del derecho pues "es posible llegar a acuerdos en el ámbito dispositivo de los derechos, pero incluso también en ámbitos no dispositivos". Esta situación se observa claramente en el ámbito de los conflictos suscitados en las crisis familiares existiendo hijos menores, pues si bien es cierto que estas materias dado el interés del menor son imperativas exigiendo siempre un pronunciamiento judicial sobre las mismas para la validez de cualquier acuerdo, sin embargo, también son materia propia de negociación. Obsérvese que recientemente se ha dictado una Sentencia que nos muestra esta tendencia. Nos referimos a un caso donde los progenitores firman un documento privado (propuesta de convenio regulador) que no llegó a ser homologado judicialmente. Frente a la línea jurisprudencial que sostenía que existiendo menores era siempre preciso la homologación judicial para que el documento firmado tuviese plena eficacia, el Tribunal Supremo ha defendido otra postura. En concreto, la STS (Sala de lo Civil) de 15 de octubre de 2018, del Pleno señala "Los acuerdos sobre medidas relativas a hijos comunes, menores de edad, serán válidos siempre y cuando no sean contrarios al interés del menor, y con la limitación 
impuesta en el art. 1814 CC, esto es, que no cabe renunciar ni disponer del derecho del menor a la pensión de alimentos, ni puede compensarse con una deuda entre los progenitores, ni someterse condicionalmente en beneficio de los menores".

Respecto de las normas dispositivas es preciso que el estudiante comprenda su naturaleza obligatoria de segundo grado pues la norma dispositiva sirve para integrar cualquier acuerdo si no ha sido expresamente excluida y sustituida por las partes. Además, no se puede obviar que los sujetos del conflicto se encuentran en una sociedad muy informada y les puede provocar frustración si descubren tras el acuerdo alcanzado que éste se aleja de las expectativas jurídicas que tenían sin que nadie les haya informado de ello.

También se deberá profundizar en la exclusión de la ley aplicable. Como señala DÍEZ PICAZO Y GULLÓN BALLESTEROS (2006, p. 171) al abordar el tema de la exclusión de la ley aplicable caben dos opciones "excluir la ley aplicable como un bloque de reglamentación de una materia y la ley aplicable como sinónima de cada una de las reglas de ese bloque"... Añaden los autores que "Cuando se ha excluido el bloque de reglamentación y en su lugar se ha acogido otro, este último debe permitir aplicación a la materia que por él se quiere regular." Nos recuerdan ya en nota a pie de página que "La STS de 7 de junio de 1979 declara que las partes no pueden renunciar a una norma dispositiva de la relación que las une para acogerse a la especial de la misma, pese a no reunir los requisitos exigidos para ello en ésta última (en el caso de autos, un arrendamiento sometido al Código civil quieren las partes que se regule por la Ley de Arrendamientos Urbanos, aunque su objeto - un solar- no es el previsto en ella-edificaciones)'”.

Además, es preciso el conocimiento del marco legal desde el acuerdo lo que determina que el estudiante deba conocer especialmente aquellas instituciones y normas jurídicas en las que pueden encontrar acomodo los consensos. Cobra así especial transcendencia el estudio de la eficacia jurídica y ejecución del convenio regulador, transacción, el acuerdo de mediación...

\subsection{Capacidad para la gestión/resolución de los conflictos jurídico-civiles de forma no litigiosa.}

Si se pretende que los estudiantes en su futuro desarrollo profesional hagan uso de sistemas de resolución de conflictos jurídico-privados consensuales, es preciso que los conozcan pues sólo de esta forma parafraseando a SOLETO MUÑOZ (2017, p. 23) podrán "realizar una evaluación de cuál es el método más adecuado de resolución, y aconsejar en relación con su evaluación...".

Principalmente en el ámbito del derecho civil se abordará, entre otras, la mediación, la negociación, la práctica colaborativa...

La mediación ${ }^{10}$ es una forma autocompositiva de gestión de conflictos en el que las partes implicadas lo gestionan ellas mismas, con la ayuda de un mediador que no realiza

\footnotetext{
${ }^{10}$ En los distintos textos legales que regulan la mediación se contienen diversas definiciones de la misma. Seguidamente se citan algunos. En la Ley 5/2012, de 6 de julio de Mediación en Asuntos Civiles y Mercantiles se define la mediación en su art. 1. "Se entiende por mediación aquel medio de solución de controversias, cualquiera que sea su denominación, en que dos o más partes intentan voluntariamente alcanzar por sí mismas un acuerdo con la intervención de un mediador”. En el art. 2.1 de la Ley 1/2009, 27 de febrero, Reguladora de la Mediación Familiar en la Comunidad Autónoma de Andalucía “... se entiende por mediación el procedimiento extrajudicial de gestión de conflictos no violentos que puedan
} 
propuestas, pero favorece la comunicación entre los afectados. La mediación civil (y familiar) puede ser extrajudicial o intrajudicial y en ella han de respetarse una serie de principios en su desarrollo que permiten la identificación de la institución: igualdad de partes, confidencialidad, voluntariedad ${ }^{11}$, neutralidad, imparcialidad, buena fe, protección del interés del menor, de los discapacitados y de las personas con la capacidad modificada judicialmente. Si tras el procedimiento de mediación se llega a un acuerdo, éste podrá ser objeto de ejecución si se han cumplido los presupuestos exigidos por la Ley ${ }^{12}$ y en cualquier caso tendrá un "plus de obligatoriedad". Como nos indica el Magistrado JOSE LUIS UTRERA en el Fundamento Segundo de la Sentencia dictada por el Juzgado de Primera Instancia $n^{\circ} 5$ de Málaga de fecha 27 de septiembre de 2012:

"A este respecto ha de señalarse que los acuerdos alcanzados en mediación y documentados en lo que la Ley 5/2012 llama acta final (artículo 22-3) o acuerdo de mediación (artículo 23) deben ser equiparados, en el ámbito de los procesos de familia, a los convenios reguladores no ratificados o no aprobados judicialmente, siéndoles de aplicación la abundante jurisprudencia sobre la materia (STS $S^{a} 1^{a} 19-121997$ y 15-22002 por todas). No obstante, ha de tenerse presente que los acuerdos alcanzados en un proceso de mediación, como el que nos ocupa, tienen un "plus" de obligatoriedad. En efecto y aunque no sea de aplicación a este proceso, ha de recordarse que el artículo 23.3 de la precitada Ley 5/2012 en su último párrafo habla del carácter vinculante del acuerdo alcanzado en mediación. Pero sobre todo esa obligatoriedad "reforzada" vendría dada porque estaríamos ante negocios jurídicos de familia cuya elaboración se desarrolla en un entorno especialmente apto para que la expresión de la voluntad allí recogida lo haya sido sin vicio alguno, pues se desarrolla, por la intervención técnica del mediador, la voluntariedad de la participación, la igualdad en el desarrollo de los debates que llevan al consenso e incluso la posibilidad de contar con información y asesoramiento suficiente."

Junto a la mediación, la práctica colaborativa es otra forma de gestión no litigiosa de los conflictos. Como nos indica SOLETO MUÑOZ (2017, p. 34) "es la forma de

surgir entre miembros de una familia o grupo convivencial, mediante la intervención de profesionales especializados que, sin capacidad de decisión sobre el conflicto, les asistan facilitando la comunicación , el diálogo y la negociación entre ellos y ellas, al objeto de promover la toma de decisiones consensuadas en torno a dicho conflicto".

${ }^{11}$ Es de destacar que el Anteproyecto de Ley de Impulso a la Mediación de 2018, en su artículo tercero propone una reforma trascendente en relación al principio de voluntariedad que caracteriza la mediación. En concreto propone la modificación de la ley 5/2012 de 6 de julio de Mediación en asuntos civiles y mercantiles, en su art. 3«1. La mediación es voluntaria. No obstante, los interesados estarán obligados a intentarla con carácter previo al inicio de un proceso declarativo en los siguientes casos: a) Medidas que se adopten con ocasión de la declaración de nulidad del matrimonio, separación, divorcio o las relativas a la guarda y custodia de los hijos menores o alimentos reclamados por un progenitor contra el otro en nombre de los hijos menores, así como aquellas que pretendan la modificación de las medidas adoptadas con anterioridad. b) Responsabilidad por negligencia profesional. c) Sucesiones. d) División judicial de patrimonios. e) Conflictos entre socios y/o con los órganos de administración de las sociedades mercantiles. f) Reclamaciones en materia de responsabilidad extracontractual que no traigan causa de un hecho de la circulación. g) Alimentos entre parientes. h) Propiedad horizontal y comunidades de bienes.

i) Derechos reales sobre cosa ajena. j) Contratos de distribución, agencia, franquicia, suministro de bienes y servicios siempre que hayan sido objeto de negociación individual. k) Reclamaciones de cantidad inferiores a 2.000 euros entre personas físicas cuando no traigan causa de un acto de consumo. l) Defectos constructivos derivados de un contrato de arrendamiento de obra. m) Protección de los derechos al honor, intimidad o la propia imagen. n) Procesos arrendaticios que hayan de ventilarse por los cauces del juicio ordinario..."

12 Vid. arts. 25 y ss. de la Ley 5/2012, de 6 de julio de Mediación en Asuntos Civiles y Mercantiles. 
resolución de conflictos que evita completamente los Tribunales salvo para la homologación en su caso del acuerdo, y se gestiona a través de reuniones en las que las partes, asesoradas por sus abogados, intentan llegar a un acuerdo". Esta práctica a diferencia de lo que ocurre en otros países (Reino Unido, Canadá...) no tiene un marco jurídico en España, lo que no impide el recurso a la misma. Como nos recuerda la autora antes citada (2017, p. 169) “...el Derecho Colaborativo supone un nuevo paradigma en el ejercicio profesional del Derecho, donde prima la cooperación entre las partes y sus abogados en aras a la búsqueda consensuada de una solución integral al conflicto que satisfaga los intereses y verdaderas necesidades de las partes, reservando la vía judicial para otorgar eficacia al acuerdo, o bien, para cuando el procedimiento colaborativo no sea el adecuado o no se haya conseguido un resultado positivo por éste". Manifiesta SOLETO MUÑOZ (2017, p. 172) que son tres las características que más identifican la práctica colaborativa "la colaboración entre las partes y sus abogados, la exclusión de la vía judicial y trabajo en red con otros profesionales colaborativos" Y merece ser destacado que "conforme a lo estipulado en los acuerdos entre clientes y abogados que se firman en estos casos, si no fuera posible la consecución del acuerdo fuera ineludible el acceso contencioso a los tribunales, el abogado participante hasta el momento cesaría en su función..."

Aun siendo conscientes que se podrían mencionar más formas de gestión no litigiosa de los conflictos, vamos a finalizar este apartado haciendo referencia a la negociación. Es imprescindible que el estudiante sea conocedor al menos de las cuestiones más básicas de la negociación a fin de que pueda ponerlas en práctica y se estimule su deseo de conocer esta materia.

Nos clarifica MANARET (2001, pp. 16) citando a JIM SEBEMIU Y DAVIX LAX en su libro The manager a negotiator que "Negociar es un proceso de interacción potencialmente beneficioso, por el que dos o más partes con algún conflicto potencial o no, buscan mejorar sus opciones de negociación a través de acciones decididas conjuntamente". Como continúa señalando el citado autor (2001, p. 17 y ss.) existen tres tipos de negociadores, el duro que pretende la victoria a cualquier precio, el suave que considera que es posible negociar de forma que todas las partes ganen y el negociador por principios que pretende llegar a un acuerdo y para ello intenta separar a las personas de su problema, centrándose en los intereses de las personas y no en sus posiciones.

El estudiante debe conocer que existen diversas estrategias de negociación que pueden conducir a una gestión constructiva de los conflictos. Sin afán exhaustivo, algunas son señaladas por MARTÍNZ SELVA (2008, pp. 420 y ss.). El compromiso y regateo donde los sujetos en disputa hacen concesiones para aproximarse a un punto en común. La negociación cooperativa en la que "ambas partes confían mutuamente y se revelan información buscando juntos una solución que les satisface". La negociación por principios donde "el punto central de la estrategia son los intereses de cada parte a los que debe satisfacer el proceso de negociación, y sobre los que debe de descansar las discusiones. Siempre que sea posible, los negociadores deben buscar beneficios mutuos... El intentar entender las razones e intereses del otro ayuda, por una parte, a la comunicación y, por otra, facilita la presentación de las propias ofertas que adquieren así un aspecto más meditado". 
En cualquier caso, para gestionar adecuadamente estos sistemas es necesario que el estudiante se familiarice con los términos valor ZOPA, MAAN ${ }^{13}$, intereses, posiciones, pues les auxiliarán en la tarea de gestión constructiva de los conflictos.

\subsection{Capacidad de adoptar soluciones creativas.}

Las normas jurídicas no responden definitiva y unívocamente a los conflictos. Como señala SÁNCHEZ HERNÁNDEZ (2015, p.478) existen dos razones que lo justifican "En primer lugar, porque las normas no constituyen un modelo acabado capaz de dar una única solución dado el carácter multívoco del lenguaje. En segundo lugar, la realidad social y los conflictos que se producen en su seno presentan múltiples matices que no pueden ser contemplados en la elaboración de la norma".

Sin embargo, ésta no es la percepción del Derecho que tiene numerosos aplicadores del mismo, proliferando la idea de que frente a un conflicto solo cabe una vía de resolución que implique ajustarse a lo específica y directamente señalado en la norma sin abrir el camino a construir otras formas de resolución que siendo legales sin embargo precisan de una visión creativa del sistema jurídico y que pueda favorecer una resolución pacífica del conflicto.

Es imprescindible promover la creatividad de los estudiantes en la gestión y en su caso resolución de los conflictos dentro del marco jurídico. Frente a la idea de que para gestionar un conflicto solo cabe la utilización de una metodología litigiosa, es preciso trabajar la posibilidad de que existan otras formas de afrontarlo en el que todos los sujetos implicados puedan ganar, aunque con metas diferentes a las que en principio se plantearon.

\section{La simulación como estrategia docente para favorecer la gestión consensual de los conflictos en las asignaturas de derecho civil.}

Una vez perfiladas las competencias que debe tener el estudiante, es preciso definir el método docente que conduce a su adquisición. Como señala DE MIGUEL DIZ (2005, p. 46) el método docente es "un conjunto de decisiones sobre los procedimientos a emprender y sobre los recursos a utilizar en las distintas fases de un plan de acción que, organizados y secuenciados, coherentemente con los objetivos pretendidos en cada uno de los momentos del proceso, nos permiten dar una respuesta a la finalidad última de la tarea educativa."

La especial naturaleza del objetivo perseguido: "capacitar al estudiante en la gestión positiva, colaborativa y consensuada de los conflictos", exige adoptar un método docente que implique activamente al estudiante y le haga aprehender estos sistemas consensuales de resolución de conflictos. Se trata de conseguir un "aprendizaje significativo" en los términos expresados por FERNÁNDEZ CANO (2009 p. 1829) que aclara "si el alumno consigue establecer relaciones sustantivas y no arbitrarias entre el

\footnotetext{
${ }^{13}$ Indica SOLETO (2017, p. 41) que "El precio de reserva, reserva o precio de abandono es la cantidad mínima de venta para el vendedor y el precio máximo de compra para el comprador... La zona de posible acuerdo (ZOPA), de existir, se encuentra entre los dos precios de reserva de las partes, y supondría que las dos partes pueden tener posibilidades de llegar a un acuerdo... el acrónimo BATNA, o MAAN en español; "best alternative to a negociate agreement", es la mejor alternativa a un acuerdo negociado. La alternativa es lo mejor que se puede hacer fuera de la actual negociación, y se diferencia de la opciones en que se encuentra fuera de la mesa de negociación, es un resultado que se puede obtener sin la participación del otro negociador".
} 
nuevo material del aprendizaje y sus conocimientos previos, es decir, si lo integra en su estructura cognoscitiva, será capaz de atribuirle significados, de construirse una representación mental del mismo y, en consecuencia, habrá llevado a cabo un aprendizaje significativo". Y este aprendizaje significativo le permitirá en el futuro incorporar las estrategias de la resolución no litigiosa de los conflictos como una forma posible de abordaje de los mismos.

En este ámbito es trascendental emplear estrategias propias del aprendizaje colaborativo pues el propio instrumento utilizado favorecerá la adquisición de las competencias dado que los estudiantes deberán gestionar sus propios conflictos y llegar a acuerdos para desarrollar la actividad. Como afirman BARKLEY et al. (2012, p.18) "El aprendizaje colaborativo... consiste en que dos o más estudiantes trabajen juntos y compartan equitativamente la carga de trabajo mientras progresan hacia los resultados de aprendizaje previstos". En este aprendizaje como continúan los autores citados "En vez. de dar por supuesto que el saber existe en algún lugar de la realidad exterior y que espera ser descubierto por el saber humano, el aprendizaje colaborativo en su definición más estricta, parte de que la base del saber se produce socialmente por consenso entre compañeros versados en la cuestión".

Nos ilustra BARKLEY et al. (2012, p. 22) que, aunque “... como profesores nos gustaría creer que podemos "contar" a los estudiantes lo que hemos aprendido, y, de ese modo transferirse de forma eficiente y precisa, es patente que no podemos transferir nuestros conocimientos preparados ad hoc a las mentes de los alumnos". Esta afirmación es especialmente relevante en el ámbito que nos ocupa pues solo si el estudiante interioriza esta opción referida a la resolución positiva y consensuada de los conflictos, podrá plantearla en su futuro desarrollo profesional. Sólo se puede interiorizar "haciendo y comprobando" sus posibles beneficios y la simulación es un instrumento idóneo para conseguirlo como una de las posibles manifestaciones del "método del caso".

Indica un estudio realizado por la Universidad Politécnica de Madrid ${ }^{14}$ que "El Método del Caso (MdC), denominado también análisis o estudio de casos, como técnica de aprendizaje tuvo su origen en la Universidad de Harvard (aproximadamente en 1914), con el fin de que los estudiantes de Derecho, en el aprendizaje de las leyes, se enfrentaran a situaciones reales y tuvieran que tomar decisiones, valorar actuaciones, emitir juicios fundamentados, etc....es una técnica de aprendizaje activa, centrada en la investigación del estudiante sobre un problema real y específico que ayuda al alumno a adquirir la base para un estudio inductivo (Boehrer, y Linsky, 1990). Parte de la definición de un caso concreto para que el alumno sea capaz de comprender, de conocer y de analizar todo el contexto y las variables que intervienen en el caso".

En el citado estudio ${ }^{15}$, basándose en los estudios de MARTÍNEZ Y MUSITO se distinguen como modelo del método del caso "casos centrados en el estudio de descripciones... casos de resolución de problemas...y casos centrados en los principios". La simulación se encontraría integrada en los casos de resolución de problemas donde "además de buscar que los alumnos analicen las variables y el contexto que intervienen en la situación se pretende que formen parte activa del desarrollo del caso, dramatizando y representándolo. Además de intentar conocer qué

\footnotetext{
${ }^{14}$ Universidad Politécnica de Madrid. Servicio de Innovación Educativa (2008), El Método del caso. Guías rápidas sobre nuevas metodologías. https://innovacioneducativa.upm.es/guias/MdC-guia.pdf. p. 4

${ }^{15}$ Universidad Politécnica de Madrid, cit., se basa en el trabajo: MARTÍNEZ, A. y MUSITU, G. (eds.). (1995). El estudio de casos para profesionales de la acción social. Madrid: Narcea, p. 11
} 
ocurre en dicha situación, serán los protagonistas de la misma convirtiéndose en los personajes de la narración”. Esta dramatización, representación permitirá a los estudiantes un aprendizaje significativo de las formas consensuales de gestión de conflictos.

Por todo ello, a fin de que el estudiante tenga presente la gestión positiva de los conflictos y se motive en ampliar conocimientos en esta materia opinamos trascendente la utilización de la estrategia docente de la simulación pues con ella podrá intuir los beneficios que esta técnica puede tener, y, desarrollar las competencias que se proponen. Como señala FACH GÓMEZ y RENGEL (2013, p. 36) "la simulación debería ser un componente importante de la educación legal, ya que es una herramienta educativa que combina el aprendizaje de la parte teórica de un caso con una acertada visión práctica del mismo. Esta combinación convierte a esta experiencia en particularmente atractiva en el marco del actual EEES dado que implica un desarrollo intenso de determinadas competencias o habilidades"

Ahora bien, no podemos obviar que, como nos indican SALAS PEREA y ARDANZA ZULUETA (1995), aunque "el empleo de la simulación permite acelerar el proceso de aprendizaje y contribuye a elevar su calidad. No puede constituir un elemento aislado del proceso docente, sino un factor integrador, sistémico y ordenado de dicho proceso. Su utilización debe tener una concatenación lógica dentro del Plan Calendario de la Asignatura". Por ello consideramos que la actividad de simulación que se propone debe ir acompañada de otras actividades formativas previas que permitiesen al estudiante obtener el máximo rendimiento de la actividad propuesta.

\subsection{Selección del conflicto.}

Dado que esta actividad de simulación se diseña en el marco de los planes de estudio del Grado en Derecho la primera cuestión que debemos plantearnos es la elección del conflicto regulado por normas civiles que sea especialmente idóneo para la gestión no litigiosa.

Aunque en principio cualquier conflicto civil podría ser susceptible de negociación consensuada, sin embargo, hay que destacar que determinados conflictos son especialmente aptos para ello. Nos referimos a aquellos que anteriormente citados que figuran en la Guía para la práctica de la mediación intrajudicial publicada por el Consejo General del Poder Judicial ${ }^{16}$. Se trataría de conflictos cuya idiosincrasia en

\footnotetext{
16 Textualmente se señalan en la Guía para la Práctica de la Mediación intrajudicial citada en nota $n^{\circ} 9$, los siguientes conflictos: "2. SELECCIÓN POR TIPO DE CONFLICTO.2.1 Cuando las partes mantengan lazos familiares o se perciba una evidente carga emocional, cualquiera que sea la pretensión jurídica formulada. a) sucesiones. b) particiones hereditarias o división de bienes comunes. c) reclamaciones entre familiares. d) alimentos entre parientes. e) relaciones entre miembros de una pareja, problemas derivados de segundas parejas de ascendientes o descendientes. f) gestión de empresas familiares. g) consecuencias patrimoniales de las rupturas de matrimonios o parejas. h) organismos tutelares y gestión de las tutelas. 2.2 Cuando el conflicto surja en una relación continuada de las partes. a) relaciones de vecindad, inmisiones, medianería, servidumbres. b) propiedad horizontal. c) comunidades de bienes. d) relación entre participes y/o órganos de administración de sociedades. e) ruptura de relaciones de colaboración empresarial (franquicias, agencia, distribución, etc.). f) derivadas del contrato de seguro. 2.3 Cuando la tutela del derecho suponga un coste no reembolsable (superior a la reclamación). a) reclamaciones de consumidores frente al empresario. b) reclamaciones de escasa cuantía. 2.4 Todos aquellos en que existan entre las partes diversos litigios o se advierta que tras el actual seguirán otros. a) posibles acciones de repetición. b) incumplimientos contractuales a consecuencia de acciones dañosas de terceros. c) reclamaciones frente a empresas insolventes. d) diversas reclamaciones frente a una misma persona o conjunto de personas. 2.5 Cuando el cauce procesal aboque a un resultado de pérdida para
} 
función a sus sujetos o circunstancias hacen adivinar que ineficiencia de la respuesta impuesta por un tercero para gestionarlo adecuadamente.

\subsection{Actividades formativas presenciales $y$ no presenciales previas a la simulación.}

Si se pretende que la actividad de simulación tenga eficacia, es preciso que el estudiante se encuentre preparado para su desarrollo. Es cierto que se "aprende haciendo" pero hay que "conocer qué se hace y por qué se hace". Por ello con carácter previo a la realización de la simulación en clase, el estudiante debe haber adquirido las competencias idóneas para que dicha actividad sea enriquecedora.

Para ello se proponen las siguientes actividades docentes previas al inicio de la simulación que tienen diversos objetivos y hacen uso de métodos de aprendizaje diferentes.

ambas partes. a) ejecuciones hipotecarias. b) ejecuciones en general. c) responsabilidad civil por nuevas construcciones (LOE). 2.6 Cuando la decisión no permita adaptarse a los matices del caso, a las circunstancias personales o comporte una percepción subjetiva de difícil aprehensión por el Juzgador. a) imposibilidad de fijar plazos, condiciones, garantías o sustitución de la prestación incumplida. b) supuestos de insolvencia. c) derecho al honor, la intimidad o propia imagen. 2.7 Si existe un alto nivel de incertidumbre en el resultado económico pretendido. a) responsabilidad civil extracontractual b) responsabilidad civil medica c) responsabilidad civil profesional, en general d) reclamaciones por cumplimiento defectuoso, de base técnica e) litigios cuya sentencia sea irrecurrible. $f$ ) cuando alguna de las partes lo haya indicado en sus propios escritos de alegaciones." 


\begin{tabular}{|c|c|c|}
\hline $\begin{array}{l}\text { COMPETENCIAS } \\
\text { Y MÉTODO }\end{array}$ & $\begin{array}{l}\text { ACTIVIDADES PREVIAS A LA } \\
\text { SIMULACIÓN }\end{array}$ & ANTECEDENTES \\
\hline $\begin{array}{l}\text { Desarrollar la } \\
\text { capacidad de } \\
\text { comprensión del } \\
\text { conflicto y la gestión } \\
\text { de conflictos de forma } \\
\text { no litigiosa. }\end{array}$ & $\begin{array}{l}\text { Realización por parte de los } \\
\text { estudiantes de un WIKI }{ }^{17} \text { en el } \\
\text { Campus Virtual donde elaboren } \\
\text { de forma cooperativa dos } \\
\text { documentos con los conceptos } \\
\text { más significativos sobre la } \\
\text { negociación y el conflicto. }\end{array}$ & $\begin{array}{l}\text { En el Campus Virtual se } \\
\text { publicarán materiales, reseñas } \\
\text { bibliográficas y/o enlaces en } \\
\text { los que el estudiante pueda } \\
\text { acceder a conocimientos } \\
\text { básicos sobre conflicto y } \\
\text { negociación. }\end{array}$ \\
\hline $\begin{array}{l}\text { Aprendizaje } \\
\text { autónomo, activo y } \\
\text { colaborativo }\end{array}$ & $\begin{array}{l}\text { Exposición de los estudiantes en } \\
\text { clase de los conocimientos } \\
\text { adquiridos en negociación y } \\
\text { conflicto. }\end{array}$ & $\begin{array}{l}\text { Favorecer el estudio de la } \\
\text { materia mediante tutorías } \\
\text { personalizadas a los grupos } \\
\text { interesados. }\end{array}$ \\
\hline $\begin{array}{l}\text { Conocimiento del } \\
\text { marco legal del } \\
\text { conflicto que permita } \\
\text { la gestión positiva del } \\
\text { mismo } \\
\text { Aprendizaje } \\
\text { autónomo, activo y } \\
\text { colaborativo junto a } \\
\text { lección expositiva }\end{array}$ & $\begin{array}{l}\text { En los ejercicios prácticos que los } \\
\text { estudiantes realicen de los } \\
\text { contenidos de la asignatura se } \\
\text { incluirán preguntas concretas acerca } \\
\text { de las diversas formas de gestionar } \\
\text { los conflictos y las normas que } \\
\text { pueden favorecer o no la adopción de } \\
\text { acuerdos. } \\
\text { Exposición oral de las mismas y } \\
\text { debate. }\end{array}$ & $\begin{array}{l}\text { Lección expositiva sobre el } \\
\text { régimen jurídico aplicable al } \\
\text { conflicto y formas de gestionar el } \\
\text { conflicto. } \\
\text { Materiales para el estudio teórico } \\
\text { del marco jurídico que afecta al } \\
\text { conflicto y sistemas de gestión. } \\
\text { Visualización de videos sobre } \\
\text { resolución alternativa de } \\
\text { conflictos (mediación...) }\end{array}$ \\
\hline
\end{tabular}

Fuente: Tabla de elaboración propia.

\subsection{Simulación.}

La simulación es una estrategia de aprendizaje en la que los estudiantes ante una situación profesional planteada actúan por ellos mismos y adoptan las decisiones más idóneas para gestionar la situación en la que se encuentran. Como nos recuerda ANDREU ANDRÉS et al. (2005, p. 1) "Para ello cuentan con un escenario, pero la decisión final siempre recae en sus manos. De hecho, la diferencia fundamental entre un juego de rol o ejercicio de dramatización, un juego y una simulación radica en que los dos primeros casos son de final cerrado... Por el contrario, las simulaciones son tareas de final abierto puro donde los participantes siempre deciden y negocian al

\footnotetext{
${ }^{17}$ Nos clarifican LÓPEZ GARCÍA y SOUVIRON MORENILLA (2010, p. 155) que "Un "Wiki” es un método rápido para crear documentos en grupo, es una colección de páginas web que pueden ser fácilmente editadas por cualquier persona, en cualquier momento y desde cualquier lugar (el ejemplo más popular es "Wikipedia”). No hay por lo general ningún redactor jefe, nadie que tenga individualmente la responsabilidad editorial final. Un "Wiki" comienza con una primera página web y cada autor, cada alumno o alumna, puede modificar esa página o añadir otras páginas al "Wiki”" simplemente creando un enlace a una página que aún no ha sido creada. Conforme se va modificando el "Wiki", las versiones antiguas de cada página se quedan guardadas y pueden ser vistas al consultar la página historial”.
} 
final". Pero también es preciso tener presente que las estrategias citadas tienen un punto en común, se basa en un método de aprendizaje por la experiencia ${ }^{18}$.

En la actividad concreta de simulación que se propone, se trata de que cada grupo de estudiantes asuma la dirección técnica de los sujetos del conflicto en función del rol que se les haya asignado. Existirán los siguientes roles:

- Letrados-as en defensa de intereses litigiosos de los sujetos en conflicto.

- Letrados-as en defensa de intereses favorecedores del consenso de cada uno de los sujetos implicados.

- Jueces-as que deben resolver el asunto planteado

La secuenciación temporal que se propone es la siguiente:

- Se formarán grupos de estudiantes, antes del inicio de la simulación (dos semanas) y se asignarán mediante sorteo los roles a cada grupo.

- El conflicto que será objeto de la simulación será publicado en el campus virtual con antelación suficiente al día señalado para poner en práctica la actividad. Se pretende favorecer que los estudiantes integrantes de cada grupo puedan preparase para realizar la actividad en función de su rol. Cada grupo elaborará un dictamen en función de su rol con la finalidad de ser entregado.

- La simulación se desarrollará en el aula en una sesión de dos horas conforme al siguiente esquema:

- Entrega de los dictámenes realizados en función del rol asignado.

- Se sortearán entre los grupos constituidos quienes van a materializar la actividad de simulación. En concreto se seleccionarán dos grupos que asuman posiciones no confrontativas referidos a sujetos diferentes, dos grupos que adoptarán posiciones litigiosas también referidas a sujetos diferentes y un quinto grupo que actuará a modo de juez-a.

- Los grupos que asuman la posición consensual simularán en el aula la gestión del conflicto que propicie un acuerdo conforme a los intereses de las partes (negociación, mediación, práctica colaborativa...).

- Los grupos que asuman la función litigiosa expondrá sus argumentos de parte en términos similares a los que se realizarían en una demanda y contestación a la misma. El grupo que asuma el rol de "juez" tras una breve reflexión adoptará una decisión sobre el asunto planteado.

- La finalización de esta actividad de simulación implicará abrir un debate general sobre los distintos métodos de resolución de los conflictos, las distintas formas de resolverlo y la valoración de las consecuencias de las diferentes estrategias utilizadas en relación a la gestión del conflicto.

En la actividad de simulación propuesta, aunque se pondrán en práctica todas las competencias que hemos señalado como necesarias en el apartado segundo de este trabajo, sin embargo, se desarrollará especialmente la capacidad para adoptar soluciones creativas en el marco de los intereses de las partes y de la normativa aplicable.

\footnotetext{
${ }^{18}$ Señala LÓPEZ BARBERÁ et al. (2000, p. 29) que "el "role-playing es un método de aprendizaje a través de la experiencia".
} 


\subsection{Evaluación.}

Respecto a la evaluación de la actividad de simulación, se hace preciso distinguir entre la evaluación de la actividad para los estudiantes y la evaluación de esta actividad en relación a la consecución del objetivo perseguido.

"El efecto motivador que la evaluación tiene en los alumnos es claro" como nos recuerda LÓPEZ ALVAREZ (2009, p. 356). Por ello si queremos dar un valor relevante a la simulación tendremos que determinar claramente los criterios de evaluación y además valorarla significativamente en relación a las otras actividades prácticas realizadas durante el curso, con base en los siguientes criterios:

- Trabajos individuales y en grupo realizados con carácter previo a la simulación y durante la misma. $0,25 \%$

- $\quad$ Dictamen. 0,25\%

- Intervención en la simulación, exposiciones y debates. 0,5\%

RÚBRICA PARA LA ACTIVIDAD DE SIMULACIÓN

\begin{tabular}{|l|l|l|l|l|}
\hline & SOBRESALIENTE & NOTABLE & MEJORABLE & NO SUPERA \\
\hline $\begin{array}{l}\text { Comprensión de } \\
\text { la naturaleza del } \\
\text { conflicto }\end{array}$ & $\begin{array}{l}\text { Plena comprensión } \\
\text { del conflicto en } \\
\text { relación a la } \\
\text { estrategia utilizada }\end{array}$ & $\begin{array}{l}\text { Sólo se tienen } \\
\text { presente algunas } \\
\text { de las } \\
\text { singularidades } \\
\text { del conflicto al } \\
\text { establecer las } \\
\text { estrategias }\end{array}$ & $\begin{array}{l}\text { Se deja } \\
\text { constancia de la } \\
\text { peculiaridad del } \\
\text { conflicto, pero no } \\
\text { se manifiesta } \\
\text { como relevante en } \\
\text { la actividad }\end{array}$ & $\begin{array}{l}\text { No se atiende a la } \\
\text { naturaleza del } \\
\text { conflicto }\end{array}$ \\
\hline $\begin{array}{l}\text { Normas jurídicas } \\
\text { utilizadas }\end{array}$ & $\begin{array}{l}\text { Conoce } \\
\text { perfectamente las } \\
\text { normas jurídicas y } \\
\text { doctrina aplicable }\end{array}$ & $\begin{array}{l}\text { Refiere algunas } \\
\text { je las normas } \\
\text { jurídicas y } \\
\text { doctrina aplicable }\end{array}$ & $\begin{array}{l}\text { Se mencionan } \\
\text { algunas normas y } \\
\text { doctrina, pero sin } \\
\text { tener relevancia } \\
\text { en el desarrollo } \\
\text { de la actividad }\end{array}$ & $\begin{array}{l}\text { No se mencionan } \\
\text { normas y } \\
\text { doctrina relevante } \\
\text { para el caso }\end{array}$ \\
\hline $\begin{array}{l}\text { Estrategias de } \\
\text { gestión del } \\
\text { conflicto }\end{array}$ & $\begin{array}{l}\text { Destreza en el } \\
\text { desarrollo de las } \\
\text { estrategias de gestión } \\
\text { del conflicto }\end{array}$ & $\begin{array}{l}\text { Se desarrollan } \\
\text { adecuadamente } \\
\text { las estrategias de } \\
\text { gestión de } \\
\text { conflicto }\end{array}$ & $\begin{array}{l}\text { Se gestiona el } \\
\text { conflicto de } \\
\text { forma confusa }\end{array}$ & $\begin{array}{l}\text { No se gestiona el } \\
\text { conflicto } \\
\text { adecuadamente }\end{array}$ \\
\hline
\end{tabular}

Fuente. Tabla elaboración propia. 
RÚBRICA PARA EL DICTÁMEN, EXPOSICIÓN E INTERVENCIÓN

\begin{tabular}{|l|l|l|l|l|}
\hline & SOBRESALIENTE & NOTABLE & MEJORABLE & NO SUPERA \\
\hline $\begin{array}{l}\text { Recursos } \\
\text { utilizados }\end{array}$ & $\begin{array}{l}\text { Utiliza distintos } \\
\text { recursos jurídicos } \\
\text { para fundamentar el } \\
\text { tema }\end{array}$ & $\begin{array}{l}\text { Utiliza pocos } \\
\text { recursos o de un } \\
\text { mismo tipo para } \\
\text { fundamentar su } \\
\text { exposición }\end{array}$ & $\begin{array}{l}\text { Utiliza pocos } \\
\text { recursos para } \\
\text { apoyar sus } \\
\text { argumentos }\end{array}$ & $\begin{array}{l}\text { No utiliza recurso } \\
\text { alguno, } \\
\text { emitiendo } \\
\text { opiniones sin } \\
\text { fundamentar }\end{array}$ \\
\hline $\begin{array}{l}\text { Claridad } \\
\text { expositiva }\end{array}$ & $\begin{array}{l}\text { Se entiende } \\
\text { fácilmente los } \\
\text { argumentos } \\
\text { presentados }\end{array}$ & $\begin{array}{l}\text { Resulta en } \\
\text { ocasiones difícil } \\
\text { la comprensión } \\
\text { de la exposición }\end{array}$ & $\begin{array}{l}\text { La exposición } \\
\text { oral es } \\
\text { desordenada y } \\
\text { carece de } \\
\text { coherencia }\end{array}$ & $\begin{array}{l}\text { No hay una } \\
\text { argumentación } \\
\text { comprensible }\end{array}$ \\
\hline $\begin{array}{l}\text { Contestación a } \\
\text { preguntas }\end{array}$ & $\begin{array}{l}\text { Responde de forma } \\
\text { precisa a todas las } \\
\text { preguntas planteadas }\end{array}$ & $\begin{array}{l}\text { Contesta } \\
\text { correctamente } \\
\text { sólo a alguna de } \\
\text { las preguntas } \\
\text { planteadas. }\end{array}$ & $\begin{array}{l}\text { Responde } \\
\text { correctamente a } \\
\text { pocas preguntas }\end{array}$ & $\begin{array}{l}\text { No contesta a las } \\
\text { preguntas } \\
\text { realizadas o lo } \\
\text { hace } \\
\text { erróneamente }\end{array}$ \\
\hline
\end{tabular}

Fuente: Tabla elaboración propia.

La evaluación de la actividad de simulación en función de los objetivos conseguidos se realizará a través de un cuestionario que responderán individualmente los estudiantes participantes en la simulación, donde se indagará en la percepción que tienen sobre los métodos de resolución de conflictos no confrontativos y litigiosos.

\subsection{Reflexiones finales.}

La simulación es una estrategia docente especialmente idónea para que el estudiante aprenda significativamente los métodos de resolución y gestión consensual de los conflictos dentro del marco jurídico. Permite, como nos indica PEÑUELAS I REIXACH (2009, p. 65) que "los alumnos integren de forma coherente y racional lo que se les enseña con lo que saben..." Son numerosas las razones que conducen a dicha afirmación:

- Potencia entre los estudiantes la comunicación y gestión de sus propios conflictos en relación a puesta en práctica de la actividad.

- Facilita la comprensión de los conflictos y la identificación de los problemas jurídicos, dado que los estudiantes deben plantear sus estrategias de actuación partiendo de los mismos.

- Permite desarrollar y ejercitar las competencias negociadoras.

- Favorece la toma de decisiones creativas, pues la actividad no tiene fijado un resultado al que deban encaminar sus estrategias. El final está abierto

- Permite experimentar la realidad del ejercicio profesional y los beneficios que las estrategias consensuadas y constructivas de los conflictos suponen pues en la actividad propuesta se simularán tanto supuestos de gestión consensuada y constructiva de conflictos como de gestión litigiosa.

No obstante, la simulación es una estrategia que debe vencer obstáculos que han de ser tenidos en cuenta en el momento de su planteamiento:

- Precisa un significativo esfuerzo organizativo que puede plantear dificultades en su desarrollo. 
- Exige una intervención activa de los estudiantes, lo que puede suponer un inconveniente cuando tienen sobrecarga de actividades a realizar en relación a otras asignaturas que se imparten en el mismo curso.

- El desarrollo de la simulación en el primer curso del Grado en Derecho puede provocar problemas añadidos dado que el estudiante todavía no ha alcanzado un nivel de conocimientos adecuado.

En cualquier caso, la simulación tiene una trascendental importancia pues presenta los mimbres suficientes para despertar en el estudiante el interés por el conocimiento del conflicto y los sistemas de gestión constructiva, consensual y positiva de los mismos, incitándole a ampliar sus conocimientos en esta materia. Se aprende haciendo y la simulación es un medio eficaz para conseguirlo.

\section{Bibliografía.}

-ALZATE SAEZ DE HEREDIA, R. (2008) "Dinámicas del conflicto en el entorno familiar", Mediación Familiar, coord. SORIA, VILLAGRASA y ARMADANS, Barcelona, pp. 21 a 44.

-ANDREU ANDRÉS, M.A./ GARCÍA CASAS, M./ MOLLAR GARCÍA, M. (2005), "La simulación y el juego de la enseñanza-aprendizaje en la lengua extranjera", Cuadernos Cervantes XI, pp. 34-38.

-BARKLEY, E.F./ CROSS, P./ HOWELL MAJOR, C. (2012), Técnicas de aprendizaje colaborativo: manual para el profesor universitario, ( $\left.2^{\mathrm{a}} \mathrm{edic}\right)$, edit. Morata, S.L.

-DÍEZ PICAZO, L. y GULLÓN BALLESTERO, A. (2016) Sistema de Derecho civil. Volumen I. Parte General del derecho civil y personas jurídicas, $13^{\mathrm{a}}$ ed., edit. Tecnos, Madrid.

-FACH GÓMEZ, K. y RENGEL, A. (2013), "El aprendizaje a través de la simulación en el moot practice: una estrategia docente para la mejora de la formación jurídica universitaria en el marco del EEES", Revista Jurídica de Investigación e Innovación Educativa (REJIE), nº. p, pp. $23-48$.

-FERNÁNDEZ CANO, A.C., "Resolución de casos prácticos. El método del caso" en Enseñar Derecho en el siglo XXI. Una guía práctica sobre el Grado en Derecho, RODRIGUEZ ARANA MUÑOZ, J. y PALOMINO LOZANO, R., edit. AranzadiThomson-Reuters, Cizur Menor 2009, pp. 235 -252.

-LÓPEZ ÁLVAREZ, L.F. "Métodos de evaluación", en Enseñar Derecho en el siglo XXI. Una guía práctica sobre el Grado en Derecho, RODRIGUEZ ARANA MUÑOZ, J. y PALOMINO LOZANO, R., edit. Aranzadi-Thomson-Reuters, Cizur Menor 2009, pp.351-370.

-LÓPEZ BARBERÁ, E./ POBLACIÓN KNAPPE, P./ COLS, E. (2000), Introducción al role-playing pedagógico, $2^{\mathrm{a}}$ ed, edit. Desclé de Brouver. 
-LÓPEZ Y LÓPEZ, A. (1999) Prólogo a la monografía Régimen jurídico de las deudas tras la disolución de la sociedad de gananciales de BLANDINO GARRIDO, edit. Tirant lo Blanch, Valencia.

-LÓPEZ GARCÍA, M. y SOUVIRON MORENILLA, J.M. (2010), "El "wiki” como recurso para desarrollar competencias en el proceso enseñanza-aprendizaje", Revista Jurídica de Investigación e Innovación Educativa (REJIE), nº 2, pp. 149-160.

-MALARET, J. Manual de Negociación y Mediación. The Harvard Euronegotiation Project. Negociaciones empresariales eficaces para juristas y directivos de empresas (2201), $2^{a}$ edic., edit. Colex, Madrid.

-MANTILLA JÁCOME, R./ BAYONA RANGEL, C./ GUEVARA MENDOZA, MARIO/ FRIAS RUBIO, C.M. (2016), en Aprendizaje del Derecho, Colombia.

-MARTÍNEZ SELVA, J.M. (2008), Manual de comunicación persuasiva para juristas, $2^{\mathrm{a}}$ edic. edit. La Ley, Madrid.

-MESEGUER VELASCO, S. "Competencias genéricas y específicas: precisión de conceptos" en Enseñar Derecho en el siglo XXI. Una guía práctica sobre el Grado en Derecho, RODRIGUEZ ARANA MUÑOZ, J. y PALOMINO LOZANO, R. edit. Aranzadi-Thomson-Reuters, Cizur Menor 2009, pp. 89-102.

-MORENO CATENA, V. (2017), "Resolución jurídica de conflictos" en Mediación y resolución de conflictos, técnicas y ámbitos, dir. SOLETO MUÑOZ, H., edit. Tecnos, Madrid, pp. 45 a 78.

-PEÑUELAS Y REICHACH, LL. (2009), La docencia y el aprendizaje del Derecho en España. Una perspectiva de Derecho comparado, $3^{\mathrm{a}}$ edit. Marcial Pons, Barcelona.

-SALAS PEREA y ARDANZA ZULUETA (1995), "La simulación como método de enseñanza y aprendizaje” en Educación Médica Superior. V.9, nº1 La Habana,

SÁNCHEZ HERNÁNDEZ, C. (2016), "Método del caso y role-playing en el ámbito del derecho civil" en Presente y futuro de la docencia universitaria, MEMBIELA, P./ CASADO, n./CEBREIROS, M.I dirc., edit. Educación Editora, pp. 477 a 482.

-SOLETO MUÑOZ, H. (2017), El abogado colaborativo, edit. Tecnos, Madrid.

-ZACCAGNINI, J.L. (2015), "El conflicto en la mediación. La perspectiva psicológica" en Tratado de mediación en la resolución de conflictos, dir. OROZCO PARDO y MONEREO PÉREZ, edit. Tecnos, Madrid, pp. 33 a 57.

-ZUMAQUERO GIL, L. (2018) en "Los juegos de rol como estrategia de enseñanzaaprendizaje para fomentar la adquisición de competencias: una experiencia en la Titulación de Grado en Turismo", Revista Jurídica de Investigación e Innovación Educativa (REJIE), n ${ }^{\circ} .13$, pp. 63-74. 